\title{
Evaluation of sensitivity and specificity of the INECO Frontal Screening and the Frontal Assessment Battery in mild cognitive impairment
}

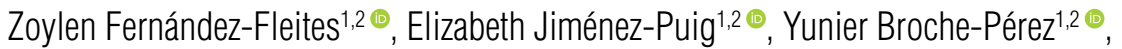 \\ Sheyla Morales-Ortiz ${ }^{1,2}$, Darlyn Alejandra Reyes Luzardo ${ }^{\circ}$, Luis Ramón Crespo-Rodríguez ${ }^{\bullet}$
}

\begin{abstract}
The Frontal Assessment Battery (FAB) and the INECO Frontal Screening (IFS) are two instruments frequently used to explore cognitive deficits in different diseases. However, studies reporting their use in patients with mild cognitive impairment (MCI) are limited. Objective: To compare the sensitivity and specificity of FAB and IFS in mild cognitive impairment (multipledomain amnestic $\mathrm{MCl}$ subtype - md-aMCI). Methods: IFS and FAB were administered to $30 \mathrm{md}-\mathrm{aMCl}$ patients and 59 healthy participants. Sensitivity and specificity were investigated using the Receiver Operating Characteristic (ROC) analysis. Results: The area under the ROC curve (AUC) of IFS for MCl patients was .82 (sensitivity $=0.96$; specificity $=0.76$ ), whereas the AUC of FAB was 0.74 (sensitivity $=0.73$; specificity $=0.70$ ). Conclusions: In comparison to FAB, IFS showed higher sensitivity and specificity for the detection of executive dysfunctions in md-aMCl subtype. The use of IFS in everyday clinical practice would allow detecting the frontal dysfunctions in $\mathrm{MCl}$ patients with greater precision, enabling the early intervention and impeding the transition to more severe cognitive alterations.
\end{abstract}

Keywords: mild cognitive impairment, cognitive assessment screening instrument, sensitivity, specificity.

\section{AVALIAÇÃO DA SENSIBILIDADE E ESPECIFICIDADE DO TESTE DE RASTREIO FRONTAL DO INECO E DA BATERIA DE AVALIAÇÃO FRONTAL NO COMPROMETIMENTO COGNITIVO LEVE}

RESUMO. A Bateria de Avaliação Frontal (FAB) e o teste de rastreio frontal do INECO (IFS) são dois instrumentos frequentemente utilizados para explorar déficits cognitivos em diferentes doenças. No entanto os estudos que relatam seu uso em pacientes com comprometimento cognitivo leve (MCI) são limitados. Objetivo: Comparar a sensibilidade e especificidade da FAB e IFS em comprometimento cognitivo leve (subtipo amnéstico de múltiplos domínios [md-aMCl]). Métodos: 0 IFS e FAB foram administrados a 30 pacientes md-aMCl e 59 participantes saudáveis. A sensibilidade e a especificidade foram exploradas usando a análise ROC. Resultados: Aárea sob a curva ROC (AUC) do IFS para pacientes com MCI foi de 0,82 (sensibilidade=0,96; especificidade=0,76), enquanto a AUC de FAB foi de 0,74 (sensibilidade=0,73; especificidade=0,70). Conclusões: Em comparação com o FAB, o IFS apresentou maior sensibilidade e especificidade para detecção de disfunções executivas no subtipo md-aMCl. 0 uso do INECO Frontal Screening (IFS) na prática clínica cotidiana, permitiria detectar com maior precisão as disfunções frontais em pacientes com deficiência cognitiva leve, possibilitando a intervenção precoce, impedindo a transição para alterações cognitivas mais graves.

Palavras-chave: disfunção cognitiva, testes de estado mental e demência, sensibilidade, especificidade.

\section{INTRODUCTION}

Wild cognitive impairment (MCI) is normal cognition and Alzheimer's disease (AD). ${ }^{1}$ The prevalence of $\mathrm{MCI}$ in people aged over 65 years ranges from 7 to $47.9 \%$, with a global prevalence of $18.9 \%$ per one thousand people. ${ }^{2} \mathrm{MCI}$ patients can be classified in two main categories: amnestic MCI (aMCI), if patients show a poor performance on

\footnotetext{
This study was conducted at the Universidad Central "Marta Abreu" de Las Villas, Santa Clara, Cuba

'Psychology Department, Universidad Central "Marta Abreu" de Las Villas - Santa Clara, Cuba. ${ }^{2}$ Cognition (Cuban Inicative on Cognitive Health) - Santa Clara, Cuba. ${ }^{3}$ Universidad Laica "Eloy Alfaro" de Manabí - Manta, Ecuador. ${ }^{4}$ Deparment of Neurology, Arnaldo Milián Castro Provincial Hospital - Santa Clara, Cuba.

Yunier Broche-Pérez. Psychology Department, Universidad Central "Marta Abreu" de Las Villas km 5 1⁄2 - ZIP 54830 Santa Clara, Villa Clara - Cuba. E-mail: yunierbp@uclv.edu.cu; yunierbroche@gmail.com

Funding: none.

Disclosure: The authors report no conflicts of interest.
}

Received on July 27, 2020. Accepted in final form on October 26, 2020.

\section{(cc) BY}


the episodic memory test, but functioning in other cognitive domains is preserved; and non-amnestic MCI (naMCI), if patients show a poor performance on cognitive evaluation covering domains other than memory such as language, visuospatial abilities, or executive functions. ${ }^{2}$ Additionally, aMCI patients could be classified in one of two possible clinical subtypes: (i) single-domain aMCI (sd-aMCI), when memory is the only impaired domain; and (ii) multiple-domain aMCI (md-aMCI), when besides the memory deficit, at least another cognitive domain is impaired (e.g., executive function, language, or visuospatial abilities). ${ }^{3}$

The annual conversion of $\mathrm{MCI}$ to $\mathrm{AD}$ is estimated between 10 and $15 \% .{ }^{4}$ Approximately $50 \%$ of people with $\mathrm{MCI}$ will be diagnosed with $\mathrm{AD}$ in the following 4 years. ${ }^{1}$. However, the specific conversion prognoses for each subtype may differ. In this sense, md-aMCI patients had more severe deficit in working memory and problem solving than sd-aMCI patients, leading to the assumption that this subtype, not pure amnestic MCIs, are at highest risk of dementia., ${ }^{5,6}$ Another study sought to more systematically and comprehensively investigate predictors of rate of cognitive decline in a longitudinal sample of individuals with $\mathrm{MCI}$, including age, genetic vulnerability, baseline cognitive performance, and baseline neuropsychiatric severity. ${ }^{7}$ The results showed that participants with composite scores for lower executive functions and greater severity of memory impairment at baseline predicted faster decline on dementia severity measures.

Recently, a meta-analytic study was conducted to explore inhibitory control (IC) in patients with aMCI, using a battery of well-validated inhibition tasks. ${ }^{8}$ According to the findings, patients with aMCI showed a generalized IC deficit, suggesting that inhibition paradigms should be routinely included in neuropsychological evaluations to obtain a more detailed overview of executive functioning in MCI patients.

Hence, early diagnosis of pathological cognitive decline becomes increasingly important in providing patients with the necessary interventions. ${ }^{9}$ Global neuropsychological batteries, such as the Montreal Cognitive Assessment (MoCA), the Mini-Mental State Examination (MMSE), and the Mattis Dementia Rating Scale second edition (DRS-2), are frequently used for neuropsychological evaluation. These screening batteries are helpful for obtaining a global cognitive performance approximation; however, they do not allow researchers to delve into executive functioning.

In this sense, the Frontal Assessment Battery (FAB) and the INECO Frontal Screening (IFS) are two frequently used instruments to explore frontal dysfunctions in different pathologies. ${ }^{10} \mathrm{FAB}$ was designed for brief investigation of executive functioning and consists of six subtests that evaluate mental flexibility, conceptualization, inhibitory control, motor programming, resistance to interference, and environmental autonomy. ${ }^{11}$ This instrument is fundamentally used for two purposes: ${ }^{12}$

1) early identification of neurodegenerative diseases, and

2) detection of executive dysfunctions in different diseases that affect the frontostriatal brain circuits.

On the other hand, IFS is a brief neuropsychological test designed to explore executive functions across neurodegenerative pathologies such as $\mathrm{AD},{ }^{13}$ behavioral variant frontotemporal dementia, ${ }^{14}$ Multiple Sclerosis (relapsing-remitting phase), ${ }^{15} \mathrm{MCI},{ }^{16}$ and $\mathrm{MCI}$ in Parkinson's disease. ${ }^{17}$ IFS is composed of eight subtests, organized into three main executive domains: ${ }^{18}$

1) inhibition and change,

2) working memory, and

3) capacity for abstraction.

Some studies have compared the clinical utility of FAB and IFS. For example, Gleichgerrcht et al. ${ }^{19}$ evaluated the usefulness of FAB and IFS in a group of 25 patients diagnosed with behavioral variant frontotemporal dementia (bvFTD) and 25 patients with AD. Compared with FAB, IFS showed a better capacity to discriminate between both dementia subtypes, a greater sensitivity and specificity for the detection of executive dysfunctions, and a high correlation with frequently used executive tests such as the Trail Making Test (part B) and the Wisconsin Card Sorting Test.

This result was confirmed by another study carried out on Peruvian patients. ${ }^{20}$ In this investigation, the diagnostic capacity of FAB and IFS was compared in a sample of 117 participants ( $35 \mathrm{AD}$ patients, 34 patients with bvFTD, and 48 healthy controls [HC]). IFS showed a greater sensitivity to discriminate between $\mathrm{AD}$ and bvFTD, compared with FAB. The objective of the present study is to compare the sensitivity and specificity of FAB and IFS in patients with multiple-domain aMCI subtype (md-aMCI). Considering previous studies that have used both instruments, the authors' hypothesis is oriented towards a better sensitivity of IFS compared with FAB.

\section{METHODS}

\section{Participants}

A total of 89 participants were evaluated: 59 cognitively healthy controls and 30 patients with $\mathrm{MCI}$ 
(multiple-domain aMCI subtype). The groups were selected according to the following criteria:

\section{Healthy control group}

The following criteria were used to select the control group: scoring more than 85 points on the Addenbrooke's Cognitive Examination Revised (ACE-R), ${ }^{21}$ no subjective complaints of memory, and preserved functioning in the activities of daily living. Psychiatric history was reported by the participants during the initial interview (conducted by an experienced psychiatrist) and an experienced neurologist performed the neurological examination.

\section{Mild cognitive impairment Group (multiple-domain aMCI subtype)}

MCI patients were classified using the criteria proposed by Petersen: ${ }^{2}$ objective impairment in formal neuropsychological measures (total score in ACE-R of at least 1.5 standard deviation [SD]below the demographically corrected mean ${ }^{21}$ and preservation of activities of daily living (Barthel index>95). ${ }^{22}$ Patients with potential causes of cognitive deficits different than neurodegenerative or cerebrovascular disease (e.g., Schizophrenia, alcoholism, epilepsy, depression, head injury) were excluded. According to the standardized neuropsychological assessment, the sample was classified as multiple-domain aMCI subtype.

Patients with MCI who showed clinical signs of depression (Geriatric Depression Scale $<5)^{23}$ or anxiety (Zung Self-Rating Depression Scale $<51$ ) were excluded from the study. ${ }^{24}$ The presence of severe sensory deficits (vision and hearing) was also considered an exclusion criterion.

\section{Instruments}

\section{Frontal Assessment Battery}

The $\mathrm{FAB}^{11}$ is a test battery easy to administer and sensitive to frontal dysfunction. FAB consists of six subtests evaluating similarities (conceptualization), motor programming, inhibitory control, verbal fluency (mental flexibility), resistance to interference, and environmental autonomy. Each subtest is scored on a maximum of three points, with a total score of 18. High scores indicate preservation of the executive functions.

\section{INECO Frontal Screening}

INECO Frontal Screening (IFS) ${ }^{14}$ is a brief neuropsychological test battery to explore executive functioning in neurodegenerative diseases. The subtests included in IFS are Luria's Fist-Edge-Palm task (three points), Conflicting instructions (sensitivity to interference) (three points), Inhibitory control (three points), Months backwards (verbal working memory) (two points), Digit Span Task (six points), Corsi Block Tapping Test (four points), Proverb interpretation (abstraction capacity) (three points), and Verbal inhibitory control (modified Hayling Test) (six points). IFS has a maximum possible score of 30 points. High scores indicate preservation of the executive functions. ${ }^{25}$

\section{Procedure and Analysis of Data}

All participants were informed of the objectives of the study and signed the informed consent form. All cognitive evaluations were done blindly ( $\mathrm{HC}$ vs $\mathrm{MCI}$ ) and independently by one neuropsychologist who applied the IFS and FAB tests to all the participants. IFS and $\mathrm{FAB}$ were applied in different sessions to reduce fatigue and potential learning effects.

Data were obtained following the regulations of the ethics committee of the Department of Psychology of Universidad Central "Marta Abreu" de Las Villas and in accordance with the Declaration of Helsinki. Data were processed using the Statistical Package for the Social Sciences (SPSS) for Windows, version 21. Descriptive statistics were used to explore participants' characteristics. An independent-sample Student's t-test was conducted to compare the executive functioning between groups. In all analyses, the homogeneity of variances was considered (by using the Levene's test for homogeneity of variances). The Cohen's $d$ effect size was calculated to estimate effect sizes in all comparisons. Values above $0.2,0.5$, and 0.8 were considered as small, medium, and large effect size, respectively. ${ }^{26}$ Linear regression was used to evaluate age and education effects over total scores of FAB and IFS. To investigate the sensitivity and specificity of FAB and IFS, the Receiver Operating Characteristic (ROC) curve analysis was performed.

\section{RESULTS}

\section{Demographics of mild cognitive impairment patients and Control Group}

The present study was conducted with 59 healthy participants and 30 patients with MCI diagnosis (multiple-domain aMCI subtype). The results of the comparison of demographics are summarized in Table 1 . There are no significant differences in age, education years, and sex between groups. Significant differences were found 
Table 1. Demographic and neuropsychological characteristics of Healthy Controls and Mild Cognitive Impairment patients.

\begin{tabular}{|c|c|c|c|c|c|}
\hline & \multicolumn{2}{|c|}{$\mathrm{HC}(n=59)$} & \multicolumn{2}{|c|}{$\operatorname{MCl}(n=30)$} & \multirow{2}{*}{ p-value } \\
\hline & M & SD & M & SD & \\
\hline Age (years) & 76.25 & 8.08 & 78.63 & 8.26 & 0.84 \\
\hline Education level (years) & 12.38 & 4.15 & 11.63 & 3.07 & 0.63 \\
\hline $\operatorname{Sex}\left(M_{a}: F\right)$ & $29-30$ & & $15-15$ & & 0.94 \\
\hline Handedness (R:L) & $59-0$ & & 29_1 & & 0.15 \\
\hline ACE-R (/100) & 89.74 & 4.36 & 68.13 & 11.01 & $<0.001$ \\
\hline $\begin{array}{l}\text { Attention and } \\
\text { Orientation (/18) }\end{array}$ & 16.67 & 1.67 & 13.96 & 2.31 & $<0.001$ \\
\hline Memory (/26) & 23.11 & 2.16 & 17.53 & 4.53 & $<0.001$ \\
\hline Verbal Fluency (/14) & 10.06 & 1.89 & 5.90 & 1.82 & $<0.001$ \\
\hline Language (/26) & 25.20 & 1.18 & 20.86 & 3.47 & $<0.001$ \\
\hline Visuospatial (/16) & 14.61 & 1.48 & 10.03 & 2.89 & $<0.001$ \\
\hline
\end{tabular}

between groups regarding the Addenbrooke's Cognitive Examination Revised (ACE-R).

Overall, age had no influence on FAB ( $\mathrm{r}=-0.086$, $p=0.27)$ and IFS $(r=-0.082, p=0.18)$, whereas years of education had a positive linear influence on $\mathrm{FAB}(\mathrm{r}=0.32$, $\mathrm{p}<0.001)$ and IFS $(\mathrm{r}=0.29, \mathrm{p}=0.004)$.

\section{Subtests shared by both INECO Frontal Screening and Frontal Assessment Battery}

FAB and IFS shared three subtests: ${ }^{19}$ Luria's FistEdge-Palm task, Conflicting instructions, and Inhibitory control (Go/No-go task) (Table 2). Two subtests showed differences between groups. Resistance to interference (Conflicting instructions) showed differences between groups. The MCI group showed lower scores than the HC group. The means of the scores in the Inhibitory control (Go/No-go task) also showed significant differences between groups. Patients with $\mathrm{MCI}$ performed worse than healthy controls. No differences between $\mathrm{HC}$ group and MCI patients were found in Luria's Fist-Edge-Palm task.

\section{Frontal Assessment Battery}

The results of $\mathrm{HC}$ participants and $\mathrm{MCI}$ patients concerning the FAB test is summarized in Table 2. In addition to the test shared by both instruments, the subtests Conceptualization, Mental flexibility, and Total score differed between groups. In all cases, MCI patients showed lower scores than HC participants. In Conceptualization and Mental Flexibility subtests,
Table 2. Performance of healthy controls and mild cognitive impairment patients in the Frontal Assessment Battery.

\begin{tabular}{|c|c|c|c|c|c|}
\hline \multirow[t]{2}{*}{ FAB subtests } & $\begin{array}{c}\mathrm{HC} \\
(\mathrm{n}=59)\end{array}$ & $\begin{array}{c}\text { MCl } \\
(n=30)\end{array}$ & $\mathbf{t}$ & p-value & d \\
\hline & M(SD) & M(SD) & & & \\
\hline Conceptualization & $2.29(0.76)$ & $1.83(0.74)$ & 2.66 & 0.009 & 0.61 \\
\hline Mental flexibility & $2.51(0.67)$ & $1.97(0.71)$ & 3.49 & 0.001 & 0.79 \\
\hline $\begin{array}{l}\text { Motor } \\
\text { programming* }\end{array}$ & $2.42(0.77)$ & $2.23(1.04)$ & 0.97 & 0.33 & 0.22 \\
\hline $\begin{array}{l}\text { Resistance to } \\
\text { interference* }\end{array}$ & $2.66(0.63)$ & $2.26(0.86)$ & 2.44 & 0.017 & 0.56 \\
\hline Inhibitory control* & $2.10(0.90)$ & $1.36(0.96)$ & 3.54 & 0.001 & 0.81 \\
\hline $\begin{array}{l}\text { Environmental } \\
\text { autonomy }\end{array}$ & $2.93(0.41)$ & $2.87(0.43)$ & 0.69 & 0.48 & 0.14 \\
\hline Total & $14.85(2.33)$ & $12.47(2.78)$ & 4.27 & $<0.001$ & 0.96 \\
\hline
\end{tabular}

*Subtests shared by both IFS and FAB tests. IFS: INECO Frontal Screening; FAB: Frontal Assessment Battery; $\mathrm{HC}$ : healthy controls; MCl: mild cognitive impairment patients; $\mathrm{SD}$ : standard deviation; M: mean, d: effect size.

the effect size of the differences was medium ( $\mathrm{d}>0.5)$. For Total Score in FAB, differences between means was large $(\mathrm{d}>0.8)$. No differences between MCI patients and the $\mathrm{HC}$ group were found in the Environmental Autonomy domain.

\section{INECO Frontal Screening}

The executive functioning of MCI patients and $\mathrm{HC}$ participants according to IFS is summarized in Table 3. In Backward months (Verbal working memory), Corsi Block Tapping Test (Spatial working memory), Modified Hayling Test (Verbal inhibitory control), and IFS total score showed significant differences between groups. In all cases, MCI patients showed poorer performance than $\mathrm{HC}$ participants. In verbal and spatial working memory domains, the effect size of the differences in the means was medium $(\mathrm{d}>0.5)$. For Verbal inhibitory control (Modified Hayling Test) and IFS total score, differences between means was large (d>0.8). No differences between MCI patients and the $\mathrm{HC}$ group group were found in the Working memory (Digit Span Task), and Abstraction capacity (Proverb interpretation) domains.

\section{Sensitivity and specificity of Frontal Assessment Battery and INECO Frontal Screening}

Figure 1 shows ROC curves of IFS (total score) and FAB (total score) for detecting MCI (multiple-domain aMCI subtype). The results showed that the area under the curve (AUC) of IFS for MCI 
Table 3. Performance of healthy controls and mild cognitive impairment patients in the INECO Frontal Screening.

\begin{tabular}{lccccc}
\hline \multirow{2}{*}{ IFS subtests } & $\mathbf{H C}$ & $\mathbf{M C l}$ & & & \\
\cline { 2 - 4 } & $\mathbf{( n = 5 9 )}$ & $\mathbf{( n = 3 0 )}$ & $\mathbf{t}$ & $\mathbf{p}$-value & $\mathbf{d}$ \\
\cline { 2 - 4 } & $\mathbf{M}(\mathbf{S D})$ & $\mathbf{M}(\mathbf{S D})$ & & & \\
\hline Motor series $^{*}$ & $2.42(0.77)$ & $2.23(1.04)$ & 0.97 & 0.33 & 0.22 \\
\hline $\begin{array}{l}\text { Conflicting } \\
\text { instructions }\end{array}$ & $2.66(0.63)$ & $2.26(0.86)$ & 2.44 & 0.017 & 0.56 \\
\hline Go/No-go task* & $2.10(0.90)$ & $1.36(0.96)$ & 3.54 & 0.001 & 0.81 \\
\hline Digit Span Task & $2.67(1.04)$ & $2.46(0.81)$ & 0.96 & 0.33 & 0.21 \\
\hline $\begin{array}{l}\text { Verbal working } \\
\text { memory }\end{array}$ & $1.69(0.50)$ & $1.26(0.69)$ & 3.34 & 0.001 & 0.76 \\
\hline $\begin{array}{l}\text { Spatial working } \\
\text { memory }\end{array}$ & $2.20(0.97)$ & $1.63(0.92)$ & 2.64 & 0.01 & 0.60 \\
\hline $\begin{array}{l}\text { Proverb } \\
\text { interpretation }\end{array}$ & $2.50(0.70)$ & $2.16(0.83)$ & 1.98 & 0.50 & 0.46 \\
\hline $\begin{array}{l}\text { Modified } \\
\text { Hayling Test }\end{array}$ & $4.84(1.62)$ & $2.36(1.86)$ & 6.46 & $<0.001$ & 1.42 \\
\hline Total & $21.05(4.11)$ & $15.6(4.28)$ & 5.83 & $<0.001$ & 1.32 \\
\hline
\end{tabular}

*Subtests shared by both IFS and FAB tests. IFS, INECO Frontal Screening; FAB: Frontal Assessment Battery; HC: healthy controls; MCl: mild cognitive impairment patients; SD: standard deviation; M: mean; d: effect size.

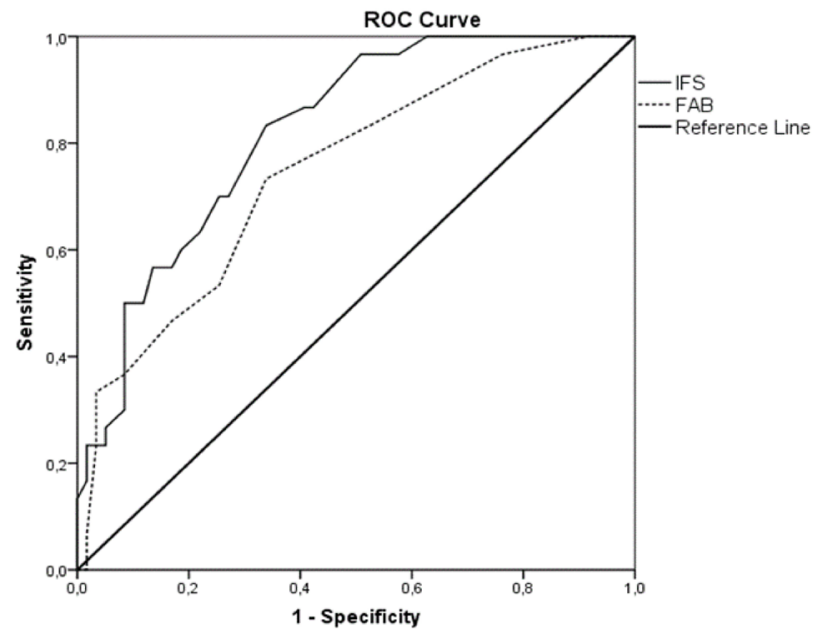

ROC: Receiver Operating Characteristic; IFS: INECO Frontal Screening; FAB: Frontal Assessment Battery.

Figure 1. Receiver Operating Characteristic curves show INECO Frontal Screening (total score) and Frontal Assessment

Battery (total score) for distinguishing between healthy controls and mild cognitive impairment patients.

patients was .82 (cutoff $=20 / 21$; sensitivity $=0.90$; specificity=0.76), whereas the AUC for FAB was 0.74 (cutoff $=13 / 14$; sensitivity $=0.96$; specificity $=0.70)($ Table 4$)$.
Table 4. Sensitivity, specificity, area under the curve, and cutoff of Frontal Assessment Battery and INECO Frontal Screening for mild cognitive impairment patients vs. healthy controls.

\begin{tabular}{lccccc}
\hline & AUC & 95\%Cl & Cutoff & Sensitivity & Specificity \\
\hline MCl vs. HC & & & & & \\
\hline IFS & 0.82 & $0.73-0.90$ & $20 / 21$ & 0.96 & 0.76 \\
\hline FAB & 0.74 & $0.64-0.85$ & $13 / 14$ & 0.90 & 0.70 \\
\hline
\end{tabular}

IFS: INECO Frontal Screening; FAB: Frontal Assessment Battery; MCI: mild cognitive impairment patients; $\mathrm{HC}$ : healthy controls; AUC: area under the curve; $95 \% \mathrm{Cl}$ : confidence interval.

\section{DISCUSSION}

The objective of the present study was to compare the sensitivity and specificity of FAB and IFS in patients with amnestic MCI multiple-domain subtype (md-aMCI). First, it was found that years of education showed a positive effect on FAB and IFS, whereas the age of participants did not show a significant effect.

The absence of the effect of age on the IFS score has been previously reported, ${ }^{18,27}$ although other authors have found opposite results. ${ }^{16}$ Thus, it is worth developing more studies aimed at verifying the effect of age on IFS scores. The present results did not show an effect of age on the total FAB score, which does not correspond to the results reported in other studies that suggest an inverse effect of age on $\mathrm{FAB}$ performance. ${ }^{28-30}$

On the other hand, a positive effect of years of education on the global and dimensional scores of FAB and IFS was verified. This result is related to some studies reporting that a higher education level has a positive influence on the performance of various tests that evaluate executive functioning. ${ }^{31-33}$ In the particular case of IFS, previous research showed significant effects of education and no significant effects for age on the scores of this instrument. ${ }^{27}$ In another study carried out with patients with dementia, compared with healthy controls, age did not show associations with IFS scores, but with years of education. ${ }^{18}$

The present findings also illustrate that IFS shows better sensitivity than the FAB for distinguishing between healthy controls and MCI patients. In this study, IFS showed higher precision compared with FAB to discriminate between MCI patients and healthy participants. In a recent study, the usefulness of IFS to discriminate between healthy controls and MCI patients was also verified..$^{16}$ The cutoff point reported by the authors is very similar to that found in our study (cutoff $=20$; sensitivity $=0.92$; specificity $=0.81$ ). 
In the specific case of Cuba, we did not find any previous study that explored the clinical utility of FAB; conversely, to date, only one study in the country has investigated the sensitivity and specificity of IFS in detecting cognitive deficits in MCI patients (md-aMCI) ${ }^{17}$ Contrary to results of the present study, the previous study showed that IFS had a low capacity for discriminating between md-aMCI patients and healthy controls. This discrepancy accounts for differences in the cognitive profiles of the MCI patients included in both studies. In the study conducted by Broche-Pérez et al. ${ }^{17}$ the md-aMCI patients did not differ from healthy controls in the following subtests: Conflicting instructions (sensitivity to interference), Months backward, and Digit Span Task (working memory). In this sense, the md-aMCI patients of the present study show a greater executive deficit, which increases the sensitivity of IFS.

It is worth noting that although the existence of executive dysfunctions in $\mathrm{MCI}$ patients has been previously published, ${ }^{34}$ the evidence for the utility of brief screening neuropsychological instruments to evaluate them is limited. ${ }^{16}$

In spite of this limitation, the results of this study are related to other research carried out on different neurodegenerative diseases, according to which a greater discriminative capacity of IFS is evidenced compared with FAB. For example, when compared with FAB, IFS has shown greater discriminatory capacity in frontotemporal dementia, ${ }^{19} \mathrm{AD}$, and behavioral variant frontotemporal dementia. ${ }^{20}$ Probably, the superior psychometric properties of IFS in comparison with FAB in the evaluation of MCI patients (md-aMCI subtype) results from the addition of subtest that had demonstrated a high sensitivity to detect subtle executive dysfunctions. ${ }^{19}$

The study had some limitations. First, the MCI group is rather small. In future studies, large samples are needed to confirm statistically significant differences between diagnostic instruments. Furthermore, future studies must delve into the effect of education on performance in FAB and IFS. It is crucial to obtain normative data on the Cuban population according to different years of education in order to prevent biased interpretations and to avoid false-positive or false-negative cases. Additionally, future research should also explore the relationship between results of cognitive screening tests and the structural and functional aspects of brain activity.

In conclusion, the present findings showed that, in comparison with FAB, the IFS presented higher sensitivity for the detection of MCI patients (md-aMCI subtype) with executive dysfunctions. We recommend the inclusion of such test in screening protocols for dementia for the early detection of executive dysfunctions in $\mathrm{MCI}$ patients, in all levels of the Cuban Public Health System. The use of IFS in everyday clinical practice would allow detecting frontal dysfunctions in $\mathrm{MCI}$ patients with greater precision, facilitating early intervention and impeding the transition to more severe cognitive dysfunctions.

\section{ACKNOWLEDGMENTS}

The authors thank Angela María Manso Gramatges for the suggestions and linguistic corrections made to the manuscript.

Authors' contributions. ZFF: conceptualization, data curation, formal analysis, methodology. EJP: conceptualization, investigation, methodology. YBP: conceptualization, data curation, formal analysis, investigation, methodology, writing - original draft. SMO: data curation, investigation, methodology, validation. LRCR: investigation, methodology, supervision.

\section{REFERENCES}

1. Hansen A, Caselli RJ, Schlosser-Covell G, Golafshar MA, Dueck AC, Woodruf BK, et al. Neuropsychological comparison of incident $\mathrm{MCl}$ and prevalent MCl. Alzheimers Dement (Amst). 2018;10:599-603. https://doi. org/10.1016/j.dadm.2018.08.009

2. Petersen RC, Caracciolo B, Brayne C, Gauthier S, Jelic V, Fratiglioni L. Mild cognitive impairment: a concept in evolution. J Intern Med. 2014;275(3):214-28. https://doi.org/10.1111/joim.12190

3. Oltra-Cucarella J, Ferrer-Cascales R, Alegret M, Gasparini R, Díaz-Ortiz LM, Ríos R, et al. Risk of progression to Alzheimer's disease for different neuropsychological Mild Cognitive Impairment subtypes: A hierarchical meta-analysis of longitudinal studies. Psychol Aging. 2018;33(7):1007-21. https://doi.org/10.1037/pag0000294

4. Roberts R, Knopman DS. Classification and Epidemiology of $\mathrm{MCl}$. Clin Geriatr Med. 2013;29(4):753-72. https://doi.org/10.1016/j. cger.2013.07.003

5. Brandt J, Aretouli E, Neijstrom E, Samek J, Manning K, Albert MS, et al. Selectivity of executive function deficits in mild cognitive impairment. Neuropsychology. 2009;23(5):607-18. https://doi.org/10.1037/a0015851

6. Jung $\mathrm{YH}$, Park S, Jang H, Cho SH, Kim SJ, Kim JP, et al. Frontal-executive dysfunction affects dementia conversion in patients with amnestic mild cognitive impairment. Sci Rep. 2020;10(1):772. https://doi.org/10.1038/ s41598-020-57525-6

7. Cerbone B, Massman PJ, Kulesz PA, Woods SP, York MK. Predictors of rate of cognitive decline in patients with amnestic mild cognitive impairment. Clin Neuropsychol. 2020:1-27. https://doi.org/10.1080/1385404 6.2020.1773933

8. Rabi R, Vasquez BP, Alain C, Hasher L, Belleville S, Anderson ND. Inhibitory Control Deficits in Individuals with Amnestic Mild Cognitive Impairment: a Meta-Analysis. Neuropsychol Rev. 2020;30(1):97-125. https://doi. org/10.1007/s11065-020-09428-6 
9. Wang B, Ou Z, Gu X, Wei C, Xu J, Shi J. Validation of the Chinese version of Addenbrooke's Cognitive Examination III for diagnosing dementia. Int J Geriatr Psychiatry. 2017;32(12):e173-9. https://doi.org/10.1002/gps.4680

10. Moreira HS, Costa AS, Castro SL, Lima CF, Vicente SG. Assessing Executive Dysfunction in Neurodegenerative Disorders: A Critical Review of Brief Neuropsychological Tools. Front Aging Neurosci. 2017;9:369. https:// doi.org/10.3389/fnagi.2017.00369

11. Dubois B, Slachevsky A, Litvan I, Pillon B. The FAB: A frontal assessment battery at bedside. Neurology. 2000;55(11):1621-6. https://doi. org/10.1212/wnl.55.11.1621

12. Kopp B, Rosser N, Tabeling S, Stürenburg HJ, de Haan B, Karnath HO, Wessel K. Performance on the Frontal Assessment Battery is sensitive to frontal lobe damage in strokepatients. BMC Neurol. 2013;13:179. https:// doi.org/10.1186/1471-2377-13-179

13. Moreira HS, Lima CF, Vicente SG. Examining executive dysfunction with the institute of cognitive neurology (INECO) frontal screening (IFS): Normative values from a healthy sample and clinical utility in Alzheimer's disease. J Alzheimers Dis. 2014;42(1):261-73. https://doi.org/10.3233/ JAD-132348

14. Torralva T, Roca M, Gleichgerrcht E, Bekinschtein T, Manes F. A Neuropsychological battery to detect specifc executive and social cognitive impairments in early frontotemporal dementia. Brain. 2009;132(Pt 5):1299309. https://doi.org/10.1093/brain/awp041

15. Bruno D, Torralva T, Marenco V, Torres-Ardilla J, Baez S, Gleichgerrcht E, et al. Utility of the INECO frontal screening (IFS) in the detection of executive dysfunction in patients with relapsing-remitting multiple sclerosis (RRMS). Neurol Sci. 2015;36(11):2035-41. https://doi.org/10.1007/s10072-0152299-6

16. Moreira HS, Costa AS, Machado A, Castro SL, Lima CF, Vicente SG. Distinguishing mild cognitive impairment from healthy aging and Alzheimer's Disease: the contribution of the INECO Frontal Screening (IFS) PLoS One. 2019;14(9):e0221873. https://doi.org/10.1371/journal. pone.0221873

17. Broche-Pérez $Y$, Bartuste-Marrero $D$, Batule-Dominguez $M$, Toledano-Toledano F. Clinical utility of the INECO Frontal Screening for detecting mild cognitive impairment in Parkinson's disease. Dement Neuropsychol. 2019;13(4):394-402. https://doi.org/10.1590/ 1980-57642018dn13-040005

18. Ihnen J, Antivilo A, Muñoz-Neira C, Slachevsky A. Chilean version of the INECO Frontal Screening (IFS-Ch). Dement Neuropsychol. 2013;7(1):40-7. https://doi.org/10.1590/S1980-57642013DN70100007

19. Gleichgerrcht $E$, Roca M, Manes F, Torralva T. Comparing the clinical usefulness of the Institute of Cognitive Neurology (INECO) Frontal Screening (IFS) and the Frontal Assessment Battery (FAB) in frontotemporal dementia. J Clin Exp Neuropsychol. 2011 Nov;33(9):997-1004. https://doi.org/10.1 080/13803395.2011.589375.

20. Custodio N, Herrera-Pérez E, Lira D, Roca M, Manes F, Báez S, et al. Evaluation of the INECO Frontal Screening and the Frontal Assessment Battery in Peruvian patients with Alzheimer's disease and behavioral variant Frontotemporal dementia. eNeurologicalSci. 2016;5:25-29. https://doi. org/10.1016/j.ensci.2016.11.001.
21. Broche-Pérez Y, López-Pujol HA. Validation of the Cuban Version of Addenbrooke's Cognitive Examination-Revised for Screening Mild Cognitive Impairment. Dement Geriatr Cogn Disord. 2017;44(5-6):320-27. https:// doi.org/10.1159/000481345.

22. Fernández-Fleites Z, Broche-Pérez Y. Moduladores psicológicos relacionados con el progreso de la capacidad funcional en pacientes afectados por accidente cerebrovascular. Fisioterapia. 2018;40(1):11-8. https://doi. org/10.1016/j.ft.2017.07.002

23. Yesavage JA. Geriatric Depression Scale. Psychopharmcol Bull. 1988;24(4):709-11.

24. Zung WW. A rating instrument for anxiety disorders. Psychosomatics. 1971;12(6):371-9. https://doi.org/10.1016/s0033-3182(71)71479-0

25. Florentino N, Gleichgerrcht E, Roca M, Cetkovich M, Manes F, Torralva T. The INECO Frontal Screening tool differentiates behavioral variant -frontotemporal dementia (bv-FTD) from major depression. Dement Neuropsychol. 2013;7(1):33-39. https://doi.org/10.1590/S198057642013DN70100006

26. Cohen J. Statistical power analysis for the behavioral Sciences. $2^{\text {nd }}$ ed. New York: Acad Press; 1988.

27. Sierra-Sanjurjo N, Belén-Saraniti A, Gleichgerrcht E, Roca M, Manes F, Torralva T. The IFS (INECO Frontal Screening) and level of education: Normative data. Appl Neuropsychol Adult. 2019;26(4):331-9. https://doi. org/10.1080/23279095.2018.1427096

28. Appollonio I, Leone M, Isella V, Piamarta F, Consoli T, Villa ML, et al. The Frontal Assessment Battery (FAB): Normative values in an Italian population sample. Neurol Sci. 2005;26(2):108-16. https://doi.org/10.1007/s10072005-0443-4

29. Lima CF, Meireles LP, Fonseca R, Castro SL, Garrett C. The frontal assessment battery (FAB) in Parkinson's disease and correlations with formal measures of executive functioning. J Neurol. 2008;255(11):1756-61. https://doi.org/10.1007/s00415-008-0024-6

30. lavarone A, Ronga B, Pellegrino L, Lore E, Vitaliano S, Galeone F, et al. The Frontal Assessment Battery (FAB): Normative data from an Italian sample and performances of patients with Alzheimer's disease and frontotemporal dementia. Funct Neurol. 2004;19(3):191-6.

31. Acevedo A, Loewenstein DA, Agrón J, Duara R. Influence of sociodemographic variables on neuropsychological test performance in Spanish-speaking older adults. J Clin Exp Neuropsychol. 2007;29(5):530-44. https:// doi.org/10.1080/13803390600814740

32. Ganguli M, Snitz BE, Lee CW, Vanderbilt J, Saxton JA, Chang CCH. Age and education effects and norms on a cognitive test battery from a population-based cohort: The Monongahela-Youghiogheny healthy aging team. Aging Ment Health. 2010;14(1):100-7. https://doi. org/10.1080/13607860903071014

33. Matute E, Leal L, Zarobozo D, Robles A, Cedillo C. Does literacy have an effect on stick constructions tasks? J Int Neuropsychol Soc. 2000;6(6):668-72. https://doi.org/10.1017/s1355617700666043

34. Huey ED, Manly JJ, Tang M-X, Schupf N, Brickman AM, Manoochehr $M$. Course and etiology of dysexecutive $\mathrm{MCl}$ in a community sample. Alzheimers Dement. 2013;9(6):632-9. https://doi.org/10.1016/j. jalz.2012.10.014 